\title{
Construção e validação do Inventário de Estilos de Temperamento do Professor (IETP)
}

\author{
Cristina Maria D’Antona Bachert \\ Solange Muglia Wechsler \\ Wagner de Lara Machado \\ Pontificia Universidade Católica de Campinas \\ Campinas, SP, Brasil
}

\begin{abstract}
Resumo
O Inventário de Estilos de Temperamento do Professor (IETP) é um instrumento de autorrelato composto por quatro dimensões: Extrovertido-Introvertido, Prático-Imaginativo, Pensamento-Sentimento e Organizado-Flexível, que teve suas propriedades psicométricas avaliadas em dois estudos. O primeiro analisou as evidências de validade baseadas nos 40 itens construídos e contou com a participação de cinco pesquisadores da área de Avaliação Psicológica. O segundo investigou as evidências baseadas na estrutura interna do IETP, que foram avaliadas por meio da Análise Fatorial Exploratória com definição de quatro fatores e seus coeficientes de fidedignidade $(\mathrm{F} 1=0,77 ; \mathrm{F} 2=0,80 ; \mathrm{F} 3=0,90 ; \mathrm{F} 4=0,70)$. Participaram deste estudo 400 professores que lecionavam no Ensino Fundamental II e Ensino Médio (média de idade=41,1 anos, SD=10,97). Uma análise de agrupamentos (Two-Step Cluster) identificou a presença de dois grupos: Catalizador $(59,9 \%)$ e Compreensivo $(40,1 \%)$. Conclui-se que o IETP possui qualidade científica para facilitar ao professor avaliar e aprimorar seu estilo pessoal de ensinar.
\end{abstract}

Palavras chave: Temperamento; Estilos; Processo ensino-aprendizagem; Formação de professores.

\section{Construct and validity od Teacher's Temperament Styles Inventory (IETP)}

\begin{abstract}
The Teacher Temperament Styles Inventory (IETP) is a self-report instrument composed of four dimensions: ExtrovertedIntroverted, Practical-Imaginative, Thinking-Feeling, and Organized-Flexible that had its psychometric properties evaluated in two studies. The first one analyzed the evidences based on 40 items designed and included five psychological assessment researchers. The second examined the evidences based on internal structure of IETP, which was assessed by Exploratory Factor Analysis with four factors set, their reliability coefficients $(F 1=0.77, F 2=0.80, F 3=0.90, F 4=0.70)$. The latter study included 400 elementary and high school teachers (Mean Age $=41.1$ years; $\mathrm{SD}=10.97$ ). The cluster analysis (Two Step Clusters) identified the existence of two profile groups discriminated between Catalyst (59.9\%) and Comprehensive (40.1\%). On conclusion, the IETP has scientific qualities in order to help teachers assess and improve their teaching style.
\end{abstract}

Keywords: Temperament; Styles; Teaching-learning process,; Teacher education.

\section{Construcción y validación del Inventario de los Estilos de Temperamento del Profesor (IETP)}

\section{Resumen}

Lo Inventario de los Estilos de Temperamento del Profesor es un instrumento de auto informe compuesto por cuatro dimensiones: Extrovertido-Introvertido, Práctico-Imaginativo, Pensamiento-Sentimiento y Organizado-Flexible que tuvieron sus propiedades psicométricas evaluadas en dos estudios. El primero examinó las evidencias de validez a partir de los ítems do IETP contó con la participación de cinco investigadores del área de Evaluación Psicológica. El segundo examinó las evidencias basada en la estructura interna de IETP, los cuales fueron evaluados por Análisis Factorial Exploratorio con la definición de cuatro factores y sus coeficientes de fiabilidad $(\mathrm{F} 1=0,77 ; \mathrm{F} 2=0,80 ; \mathrm{F} 3=0,90 ; \mathrm{F} 4=0,70)$. El estudio incluyó a 400 maestros que enseñaban en escuela básica (Media de Edad=41,1 años; $\mathrm{SD}=10,97$ ). Un análisis de clusters (Two Step Clusters) identificó la presencia de dos grupos de perfiles Catalizador (59,9\%) y Comprensivo (40,1\%). Se concluyó que la IETP tiene calidad científica para permitir la mejora del estilo de enseñanza.

Palabras clave: Temperamento; Estilos; Proceso de enseñanza y aprendizaje; Formación del profesorado. 
No início do século XX Jung apresentou sua teoria sobre os Tipos Psicológicos (Jung 2011/1921). Segundo este modelo, o tipo psicológico é definido como uma atitude habitual da pessoa onde há o predomínio da "extroversão" ou da "introversão", atitudes definidas a partir da orientação de energia psíquica, manifestada de forma consciente ou inconsciente. Considerando que há um continuum entre essas duas características, uma pessoa pode apresentar comportamentos que variem entre os dois polos (Ramos, 2002). De acordo com Jung, tanto a introversão quanto a extroversão podem destacar qualidades positivas ou negativas, possivelmente influenciadas por atributos genéticos ou fisiológicos mediados pelo ambiente (Primi, Wechsler, Nakano, Oakland \& Guzzo, 2014), e são associadas a outras funções psicológicas - pensamento, sentimento, sensação e intuição, para complementar a classificação das diferenças individuais. Jung usou o conceito criado por Galeno para explicar o mecanismo pelo qual a disposição da psique e sua função inferior passam a dominar temporariamente, causando um desequilíbrio de forças que pode levar à doença (Ramos, 2005).

Assim como Jung, Oakland definiu temperamento a partir da influência da associação de aspectos biológicos, ambientais e escolhas pessoais no comportamento de uma pessoa (Oakland, Pretorious and Lee, 2008), evidenciando uma maneira particular e característica de enfrentar as situações de vida diária (Guzzo, Riello, Primi, Serrano, Ito, \& Pinho, 2004). O temperamento é a base para o desenvolvimento da personalidade (Shinner, 2011) e tem um papel fundamental no desenvolvimento e na promoção da saúde mental de um indivíduo, bem como em seus fatores de proteção e de risco desde a infância (Bayly \& Garstein, 2013; Klein \& Linhares, 2010). De modo geral, o temperamento é caracterizado por comportamentos apresentadas de forma bipolar, como por exemplo: extrovertido-introvertido, prático-imaginativo, pensamento-sentimento, organizado-flexível (Wechsler, Benson, Oakland, \& Lourençoni, 2014).

O temperamento pode ser identificado desde o nascimento (Lima, Lemos, \& Guerra, 2010), sendo estável ao longo da vida (Canals, Hernandéz-Matinez, \& Fernandéz-Ballart, 2011). Embora indique a predisposição de ação, não há como afirmar que pessoas, acontecimentos e estímulos possam desencadear as mesmas respostas em um indivíduo (Lee, Oakland, \& Ahn, 2010; Wechsler et al., 2014). Isto porque os comportamentos resultam da integração entre a história de vida de cada ser humano e fatores que modificam o significado e a valoração dos estímulos percebidos em um determinado momento, tais como as influências ambientais, motivações e habilidades pessoais (Ito \& Guzzo, 2002; Klein \& Linhares, 2007; Pinho, 2005; Quintana \& Muñoz, 2010), não sendo vistos como um estilo permanente da pessoa. Considerando a maturação do organismo, estas alterações também podem ocorrer devido à plasticidade do cérebro em gerar ou manter conexões neurais, provocando uma mudança qualitativa do temperamento ao longo do tempo (Joyce, 2010).

O termo "estilo" foi utilizado pela primeira vez em 1937 quando Allport, tendo como referência a Teoria dos Tipos Psicológicos de Jung, definiu diferentes estilos de personalidade e formas de agir das pessoas, descrevendo padrões habituais ou formas preferenciais das pessoas para, por exemplo, pensar, aprender ou ensinar (Sternberg \& Grigorenko, 2001). Os referidos autores sinalizam que, embora este construto tenha sido modificado e ampliado nas últimas décadas, a ideia central colocada por Allport permanece praticamente a mesma até os dias de hoje.

Assim sendo, os estilos de comportamento não são permanentes, podendo ser definidos como a maneira preferencial de uma pessoa pensar e se comportar frente a determinadas situações de seu cotidiano (Weschler, 2008). Esta forma de reação previsível pode explicar como cada indivíduo interpreta e elabora suas próprias experiências com o intuito de estabelecer relações com a realidade percebida (Ávila, 2008). Wechsler (2009) ressalta que, embora indique uma predisposição para agir, estas preferências individuais podem ser modificadas de acordo com o contexto em que a pessoa se encontra.

Zhang e Sternberg (2005) adotaram a nomenclatura "Estilos Intelectuais" e, de forma sucinta, definiram estilos como o modo preferencial que as pessoas têm para processar informações (percepção, organização e análise de estímulos do ambiente), e lidar com as tarefas que lhes são destinadas nas diferentes esferas de sua vida. Sendo assim, os estilos estão associados ao modo preferencial de ação de um indivíduo (Armstrong, Peterson, \& Rayer, 2011) e não a uma habilidade mais restrita à capacidade cognitiva (Runco, 2007).

Em comum, temperamento e estilos intelectuais permitem identificar o quanto uma pessoa se ajusta ou não às demandas do ambiente ou da tarefa a ser realizada. Conforme exposto, ambos os construtos têm como objetivo a adaptação e o equilíbrio de uma pessoa. Portanto, um estilo que facilite este processo será avaliado como positivo; já quando causar dificuldades será considerado negativo (Sternberg, 1997; Zhang \& Sternberg, 2005). Considerando a capacidade humana de aprender e mudar, tanto os estilos positivos podem ser fortalecidos quanto os avaliados como negativos 
podem ser modificados por meio da aprendizagem (Pitta, Santos, Escher, \& Bariani, 2000; Sadler-Smith, 2009).

Os estilos de temperamento são constituídos a partir da fusão entre aspectos comuns do temperamento e dos estilos intelectuais acima citados, considerando também que ambos são afetados por aspectos biológicos, cognitivos, afetivos, psicológicos e sociais, bem como das escolhas pessoais como fatores que podem ser considerados na análise de uma ação realizada pela pessoa (Callueng \& Oakland, 2014). Assim sendo, para atingir o equilíbrio, condição de saúde definida por Galeno há mais de 2500 anos, será necessário aprender como relacionar os aspectos citados de forma harmoniosa. Este estado de equilíbrio está baseado na mediação entre instâncias da cognição e da personalidade (Nakano, Santos, Zavarize, Wechsler, \& Martins, 2010), que inclui também valores e crenças pessoais.

Os estilos de temperamento do professor podem ser observados no cotidiano escolar por meio das práticas de ensino e das estratégias ou técnicas de ensino que cada docente utiliza em suas aulas. Este conjunto de ações e práticas pedagógicas é definido como "estilo de ensinar", forma consistente e habitual do professor quando se relaciona com seus alunos com o objetivo de oportunizar a construção de conhecimentos (Borgobello, Peralto, \& Roselli, 2010). Pesquisas indicam que a identidade do professor é construída progressivamente, na medida em que cada docente aprende a identificar necessidades e criar estratégicas de intervenções para atendê-las, bem como relacionar novas metodologias e recursos instrucionais aos seus conhecimentos específicos e às suas experiências de vida, criando assim um significado para suas ações (Evans \& Warring, 2009; McMahon, 2006; Reali, Tancredi, \& Mizumaki, 2008).

A construção de um inventário de estilos de temperamento do professor pretende unir estilos intelectuais e de temperamento para identificar diferentes formas de reação de cada docente ao ambiente escolar que podem intervir em seu estilo de ensinar. Assim sendo, por meio do autoconhecimento, a identificação de seu estilo de temperamento docente pode oportunizar ao professor questionar os processos que utiliza para ensinar, bem como o que aprende enquanto ensina (Garcia, 1998). Este exercício pode incentivar o educador a avaliar suas concepções sobre sua prática em sala de aula, ao mesmo tempo em que procura apoio conceitual e metodológico para rever a maneira como planeja e organiza as situações de aprendizagem. Ao longo deste processo, o professor poderá ter a oportunidade de criar e ampliar seu repertório de ações que visam atender as demandas do cotidiano escolar e a superação dos obstáculos percebidos em sala de aula, compondo seu estilo de ensinar (Albuquerque, 2010; Ávila, 2008; Evans \& Warning, 2009).

Considerando a importância de que o professor possa conhecer melhor e refletir sobre a forma como reage às demandas do ambiente escolar e atua em sala de aula, os objetivos desta pesquisa foram: construir e validar um instrumento de autorrelato para identificar o temperamento do professor, aqui intitulado Inventário de Estilos de Temperamento do Professor (IETP).

\section{Método}

Esta pesquisa foi submetida ao Comitê de Ética em Pesquisas com Seres Humanos e obteve seu parecer de aprovação com registro CAAE 27977814.0.0000.5481. Cumprido este pré-requisito, foram então realizados dois estudos para a validação do IETP que serão apresentados separadamente. $\mathrm{O}$ primeiro estudo investigou as evidências de validade baseadas no conteúdo dos itens do IETP. O segundo estudo avaliou as evidências de validade baseadas na estrutura interna do Inventário.

\section{ESTUDO 1 \\ Criação e Análise Semântica dos Itens}

Participaram deste estudo cinco psicólogos. Dois deles eram mestrandos e três eram doutorandos em Psicologia, com pesquisas na área educacional e de desenvolvimento de potencial humano. Todos assinaram o Termo de Consentimento Livre e Esclarecido antes de receber o material para análise contendo as definições de cada categoria dos estilos de temperamento e uma tabela com a primeira versão dos itens criados.

\section{Instrumento: Inventário de Estilos de Temperamento do Professor (IETP)}

A construção e a validação do Inventário de Estilos de Temperamento do Professor tiveram como ponto de partida os seguintes instrumentos: Student Styles Questionnarie (Oakland, Glutting, \& Horton, 1996), e o "Inventário de Estilos de Temperamento para Adultos" (Oakland \& Wechsler, 2014). Os dois instrumentos são compostos por questões de escolha forçada e possuem os mesmos referenciais teóricos: Tipos Psicológicos de Jung (2011/1913) e MBTI - Myers-Briggs Type Indicator (Myers \& Briggs, 1990).

O Inventário de Estilos de Temperamento do Professor (IETP) foi criado utilizando situações típicas do cotidiano do professor em sala de aula. O IETP é 
TABELA 1

Dimensões e categorias do IETP

\begin{tabular}{|c|c|c|}
\hline Dimensão & Cat & egoria \\
\hline Social & \begin{tabular}{l}
\multicolumn{1}{c}{ Extrovertido $(\mathrm{E})$} \\
foco: o que acontece à sua volta \\
facilidade de relacionamento interpessoal \\
atividades em grupo \\
gosta de discutir assuntos diversificados \\
prefere expor suas ideias oralmente
\end{tabular} & $\begin{array}{l}\text { Introvertido (I) } \\
\text { foco: suas ideias e seu mundo interno } \\
\text { reservado e seletivo } \\
\text { atividades individuais } \\
\text { gosta de discutir sobre temas de interesse pessoal } \\
\text { prefere expor suas ideias por escrito }\end{array}$ \\
\hline $\begin{array}{l}\text { Processamento } \\
\text { da Informação }\end{array}$ & $\begin{array}{l}\quad \text { Prático (P) } \\
\text { fatos } \\
\text { realista e pragmático } \\
\text { valoriza informações percebidas por meio dos sentidos } \\
\text { conservador, tradicional } \\
\text { foco: presente }\end{array}$ & $\begin{array}{l}\text { Imaginativo (M) } \\
\text { ideias } \\
\text { hipóteses, possibilidades } \\
\text { associação de conceitos } \\
\text { inovador } \\
\text { foco: futuro }\end{array}$ \\
\hline $\begin{array}{l}\text { Processo } \\
\text { Decisório }\end{array}$ & $\begin{array}{l}\quad \text { Pensamento (N) } \\
\text { soluções justas e objetivas } \\
\text { crenças e opiniões reconhecidas socialmente } \\
\text { raciocínio lógico e analítico } \\
\text { relações mais formais } \\
\text { decisões baseadas em mérito }\end{array}$ & $\begin{array}{l}\quad \text { Sentimento (S) } \\
\text { coloca-se no lugar do outro } \\
\text { posições baseadas em sentimentos e vínculos afetivos } \\
\text { valoriza a harmonia } \\
\text { relações mais próximas } \\
\text { decisões baseadas no potencial e no esforço }\end{array}$ \\
\hline Planejamento & $\begin{array}{l}\qquad \text { Organizado (0) } \\
\text { valoriza a rotina } \\
\text { atividades planejadas com antecedência } \\
\text { dificuldade de lidar com imprevistos } \\
\text { material e registros organizados } \\
\text { foco nos procedimentos de uma tarefa }\end{array}$ & $\begin{array}{l}\text { Flexível (F) } \\
\text { planejamento é feito conforme a demanda } \\
\text { atividades criadas conforme a necessidade } \\
\text { facilidade para lidar com situações inesperadas } \\
\text { desorganizado } \\
\text { foco no prazer em realizar uma tarefa }\end{array}$ \\
\hline
\end{tabular}

composto por 40 itens de escolha forçada agrupados em quatro dimensões: Social (Extroversão/Introversão), Processamento da Informação (Pragmático/Imaginativo), Processo Decisório (Pensamento/Sentimento) e Planejamento (Organizado/Flexível), conforme exposto na Tabela 1.

O IETP é um instrumento de autorrelato em que os estilos de temperamento avaliados estão relacionados ao modo como cada professor responde às demandas dos processos de ensino e de aprendizagem, incluindo a forma como organiza as atividades em sala de aula (Muritiba, Casado \& Muritiba, 2010). Todos os itens são de orientação positiva, sendo que cada alternativa está relacionada à uma das categorias avaliadas. A pontuação em cada uma das quatro dimensões é feita de acordo com a opção selecionada pelo professor, compondo assim seu estilo de temperamento docente.

\section{Procedimentos}

Foram necessárias quatro fases de avaliação dos itens. A partir da tabulação de frequência de resposta dos juízes foram considerados aprovados os itens com índice de concordância entre $80 \%$ e $100 \%$ entre o parecer dos Juízes e a proposta da pesquisadora para cada uma das categorizações. Durante o processo de revisão alguns itens foram reescritos e outros descartados, sendo criados novos para substituí-los. Feita a reescrita dos itens seguia-se uma etapa de avaliação dos Juízes. Neste processo, as definições de cada uma das oito categorias também foram aprimoradas a fim de tornalas mais concisas e objetivas a partir das sugestões e críticas feitas pelos juízes. Cabe aqui ressaltar que, neste processo, alguns itens tiveram sua categorização original alterada.

\section{Resultados}

Dentre os itens aprovados pelos avaliadores, são apresentados a seguir, a título de exemplo, quatro itens - um por dimensão avaliada pelo IETP, sendo que cada alternativa representa um de seus polos. O primeiro exemplo está relacionado à Dimensão Social: "Durante o intervalo das aulas eu gosto de (A) conversar (Extrovertido); (B) ficar um pouco sozinho (Introvertido). Quanto à Dimensão Processamento da Informação: "Gosto de usar em minhas aulas estratégias (A) novas e diferentes (Imaginativo); (B) práticas e conhecidas (Prático). Como exemplo da Dimensão Processo Decisório: "Quando há um conflito durante a aula busco resolvê-lo (A) por meio da conciliação entre as partes (Sentimento); (B) usando as regras da escola 
(Pensamento). Por fim, na Dimensão Planejamento: “O que é planejado (A) deve ser cumprido (Organizado); (B) pode ser modificado (Flexível).

O Estudo 1 foi encerrado após a tabulação das categorizações propostas pelos Juízes na quarta etapa de avaliação pois os 40 itens que compõem o IETP obtiveram um índice mínimo de concordância de $80 \%$. Este resultado indica que todos os itens revistos representam as categorias avaliadas em cada uma das dimensões de temperamento definidas, condição que atesta as evidências de validade baseadas no conteúdo dos itens do IETP (Pasquali, 2007).

\section{ESTUDO 2 Análise Psicométrica do IETP}

\section{Amostra}

A amostra deste estudo foi composta por 400 professores que lecionavam no Ensino Fundamental II e Ensino Médio em escolas particulares e públicas do Estado de São Paulo. No total, 437 professores responderam ao IETP, mas 37 foram excluídos da amostra final por não terem respondido a todas as questões ou terem assinalado as duas alternativas de resposta, contrariando a orientação dada pela pesquisadora.

Dentre os participantes, $88,25 \%$ trabalhavam em escolas públicas e $11,75 \%$ em escolas particulares, sendo que $67 \%$ são mulheres e $33 \%$ homens. A idade média desta amostra é de 41,1 anos, com desvio padrão de 10,97. A maior parte dos docentes lecionava no Ensino Fundamental II e no Ensino Médio (41\%), sendo que $34 \%$ dedicavam-se exclusivamente ao Fundamental II e $25 \%$ ao Ensino Médio. Constatou-se que 39,4\% desta amostra trabalhava apenas um período do dia - manhã ou tarde; $49,3 \%$ dois períodos; $11,3 \%$ três períodos manhã, tarde e noite, cumprindo uma jornada de 15 a 18 aulas por dia (o que corresponde a uma carga horária semanal de 75 a 90 aulas, considerando um professor que trabalhe apenas de $2^{\mathrm{a}}$ feira à $6^{\underline{a}}$ feira).

Os dados referentes à formação deste grupo de professores indicam que $83,3 \%$ possuía apenas uma graduação. Deste total, $4,8 \%$ não tinham habilitação para lecionar na Educação Básica, pois são administradores de empresas, contadores, advogados, engenheiros, analistas de sistemas, jornalistas. Outros possuíam apenas cursos tecnológicos, como Metalurgia e Logística $(0,5 \%)$. Foi constatado que $16,7 \%$ dos docentes tinham uma segunda graduação. Neste grupo, composto por 67 professores, três optaram por um curso que não tem relação com a área de Educação, como Direito e Administração de Empresas. Os demais 63 fizeram um curso de licenciatura ou Pedagogia. E
$0,7 \%$ investiram em uma terceira graduação, sendo que neste grupo apenas um dos professores optou por um curso que não está relacionado à área educacional.

Tendo como base a primeira graduação dos professores como a que os habilitou a lecionar na Educação Básica, nota-se que 28,3\% das professoras possuíam licenciatura em Letras, 13,1\% em Biologia (ou Ciências Biológicas) e 10,8\% em Matemática. Já entre o grupo masculino, as licenciaturas identificadas com maior frequência foram Matemática $(15,9 \%)$ e História (12,1\%). A terceira colocação ficou dividida entre as licenciaturas de Filosofia e Geografia, com $10,6 \%$ cada.

No tocante ao tempo de experiência em sala de aula, $26,7 \%$ dos professores ingressou na profissão há menos de cinco anos. Os professores mais experientes, que atuam há mais de 10 anos, compõem $46,3 \%$ da amostra. Professores em processo de aposentadoria ou já aposentados representam $11,7 \%$ dos participantes. Nota-se também que o percentual de professores iniciantes é baixo, 3,5\%. Foi detectado nesta amostra que não existe relação entre idade e tempo de experiência em sala de aula, pois foram encontrados professores com menos de um ano de experiência com idade entre 26 e 50 anos. Já na faixa entre 1 ano e 5 anos de experiência a idade dos professores varia entre 19 anos e 60 anos.

\section{Procedimentos}

Para a coleta de dados, a pesquisadora participou de reuniões pedagógicas já previstas no calendário escolar destas instituições. De modo geral, poucos professores presentes a estas reuniões não quiseram participar da pesquisa. Destes, a maior parte permaneceu na sala fazendo atividades de registro pedagógico (correção de atividades, preenchimento de diário de classe ou relatórios solicitados pela coordenação).

O estudo de Evidências de Validade de Construto do Inventário de Estilos de Temperamento do Professor foi efetuado com o auxílio dos programas SPSS, v.21 e Factor, v.9.3 (Lorenzo-Seva \& Ferrando, 2006). Para o tratamento dos dados foram utilizadas a Correlações Tetracóricas, a Fidedignidade dos Fatores de Mislevy, a Análise Fatorial Exploratória pelo Método Unweighted Least Squares com rotação Promin. A análise de Cluster foi realizada após a Análise Fatorial com os escores fatoriais de cada escala via Método de Regressão Linear.

\section{Resultados}

A matriz de Correlações Tetracóricas indicou uma baixa correlação entre os itens, sendo indicada a exclusão de 17 itens com valores de correlação 
menor do que 0,3. Com os 23 itens restantes foi então rodada a Analise Fatorial Exploratória (Tabela 2) com quatro fatores: Extrovertido-Introvertido (F1), Prático-Imaginativo (F2), Pensamento-Sentimento (F3) e Organizado-Flexível (F4), opção feita tendo como base a construção teórica do IETP. Os quatro fatores apresentaram os respectivos autovalores: 3,65 , 1,71, 1,28 e 1,11. Juntos explicaram 40\% da variância comum dos escores. A fidedignidade dos fatores indica que a consistência interna do IETP é considerada boa, sendo $\mathrm{F} 1=0,77, \mathrm{~F} 2=0,80, \mathrm{~F} 3=0,93$ e $\mathrm{F} 4=0,70$.

Por meio da análise de clusters (ou agrupamentos) identificou-se uma estrutura de dois grupos formados por diferentes combinações dos quatro fatores do IETP. Esta solução teve como referência o índice Bayesian Information Criterion (BIC), que teve seu menor valor, isto é, menos resíduos, para dois grupos. Neste sentido, a composição dos perfis desta amostra detectou dois grupos homogêneos considerando as similaridades entre as dimensões que constituem os estilos de temperamento do professor. Conforme demonstrado na Figura 1, a configuração dos perfis resultantes do tratamento estatístico dos dados indicou que a definição do estilo de temperamento do professor foi estabelecida a partir do Fator Imaginativo, que apresentou a maior média padronizada no Cluster $1,-0,90$, indicando a categoria antagônica, Prático. A carga deste fator no Cluster 2 foi de 0,60 . Nota-se que as categorias: Imaginativo e Prático - componentes da dimensão Processamento da Informação, foram identificadas como o preditivo dos dois perfis configurados. Assim sendo, tomando como referência os escores fatoriais, o Perfil 1 (Cluster 2) representa 59,9\% da amostra e é formado pelas categorias Imaginativo (M), Pensamento (N), Flexível (F) e Extrovertido (E). O Perfil 2 (Cluster 1) representa 40,1\% da amostra, sendo composto pelas categorias Prático (P), Sentimento $(\mathrm{S})$, Organizado (O) e Introvertido (I).

TABELA 2

Carga fatorial dos Itens, Variância Explicada e Fidedignidade do IETP

\begin{tabular}{|c|c|c|c|c|c|}
\hline & Item & Fator E-I & Fator P-M & Fator N-S & Fator O-F \\
\hline \multirow{23}{*}{$\begin{array}{c}\text { Análise Fatorial } \\
\text { Exploratória }\end{array}$} & 1a_I & 0,028 & 0,449 & 0,111 & $-0,161$ \\
\hline & 5a_M & 0,450 & $-0,193$ & 0,191 & $-0,013$ \\
\hline & 6b_I & $-0,207$ & 0,503 & 0,161 & $-0,013$ \\
\hline & $8 b \_F$ & 0,043 & 0,076 & 0,015 & 0,650 \\
\hline & 9b_l & 0,054 & 0,515 & $-0,014$ & 0,117 \\
\hline & 11a_F & 0,201 & 0,092 & 0,017 & 0,269 \\
\hline & 13a_I & $-0,123$ & 0,740 & $-0,028$ & $-0,021$ \\
\hline & 14a_F & $-0,020$ & $-0,147$ & 0,123 & 0,447 \\
\hline & 18b_M & 0,530 & 0,057 & $-0,080$ & 0,13 \\
\hline & 19b_F & 0,018 & 0,083 & 0,153 & 0,474 \\
\hline & 22a_I & $-0,083$ & 0,030 & 0,395 & 0,129 \\
\hline & 23b_M & 0,680 & 0,050 & 0,086 & $-0,113$ \\
\hline & 26a_I & $-0,174$ & 0,148 & 0,410 & 0,031 \\
\hline & 29b_S & 0,088 & $-0,078$ & $-0,315$ & $-0,272$ \\
\hline & 30b_M & 0,372 & 0,040 & $-0,095$ & 0,098 \\
\hline & 31b_F & $-0,083$ & $-0,170$ & $-0,166$ & 0,456 \\
\hline & 32b_s & 0,217 & 0,131 & $-0,525$ & $-0,075$ \\
\hline & 33b_I & 0,170 & 0,756 & $-0,064$ & $-0,050$ \\
\hline & 35b_l & 0,328 & $-0,017$ & 1,004 & $-0,241$ \\
\hline & 37a_I & $-0,479$ & 0,198 & 0,070 & 0,038 \\
\hline & 38b_M & 0,590 & $-0,040$ & 0,038 & 0,009 \\
\hline & 39b_F & 0,291 & 0,016 & $-0,110$ & 0,334 \\
\hline & 40b_s & 0,065 & 0,099 & $-0,440$ & $-0,125$ \\
\hline \multicolumn{2}{|c|}{ Variância Explicada } & 2,09 & 2,08 & 2,07 & 1,51 \\
\hline \multicolumn{2}{|c|}{ Fidedignidade Explicada } & 0,77 & 0,80 & 0,93 & 0,70 \\
\hline
\end{tabular}

Categoria dos itens do IETP: E (Extrovertido), I (Introvertido), P (Prático), M (Imaginativo), N (Pensamento), $\mathrm{S}$ (Sentimento), O (Organizado), F (Flexível). 


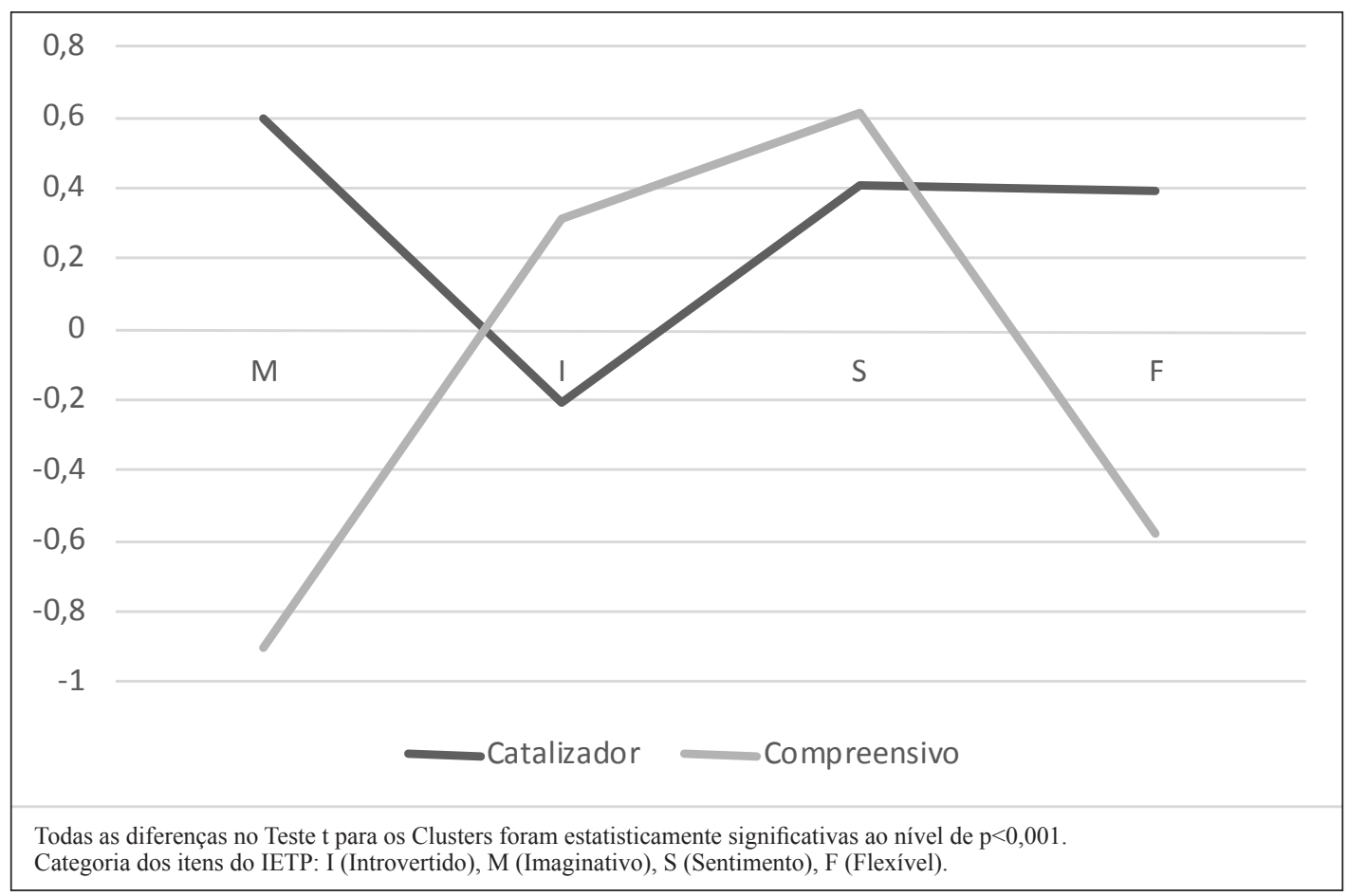

Figura 1. Média dos Perfis Catalizador e Compreensivo nos fatores I-E, P-M, N-S e O-F do IETP

O Perfil 1, denominado Catalisador, reuniu professores com tendência a buscar constantemente uma maneira diferente para ensinar algo ou utilizar os conceitos aprendidos. Esta mobilização tem como objetivo envolver o aluno em atividades que, além de necessárias também podem ser prazerosas. Neste sentido, valorizam o uso de atualidades como ferramenta de contextualização e proposição de problemas que possam favorecer aos estudantes explorar seu entorno. Segundo os professores do estilo Catalisador, a identificação e a análise de problemas cotidianos à luz dos conhecimentos aprendidos na escola podem instigar a melhoria da qualidade de vida e a construção de soluções inovadoras. Neste processo o desenvolvimento dos alunos pode ser incentivado por meio de trabalhos em pequenos grupos que podem envolver também situações para aprender a trabalhar de forma colaborativa. A competição pela busca da melhor solução é estimulada entre os grupos de alunos com o intuito de motivá-los e exercitar o trabalho em equipe. Nos momentos em que é preciso posicionar-se, busca decidir da forma mais justa e objetiva possível, usando como referência o desempenho de cada aluno e as regras da escola.

Professores com perfil Catalisador apresentam facilidade para lidar com imprevistos. Seus planos de ensino são dimensionados a partir do interesse dos alunos; as avaliações e trabalhos são definidos conforme o andamento das aulas e o desempenho dos alunos. As modificações realizadas no planejamento inicial podem incorrer em atrasos no cumprimento do que foi programado institucionalmente. Considerando que o foco de atenção do professor Catalisador é envolver o aluno e criar condições para que ele possa aprender, há risco de que a documentação pedagógica se encontre desatualizada, o que parece não incomodar ao professor com este estilo de temperamento. Embora tenha facilidade para estabelecer novos relacionamentos pessoais, professores com perfil Catalisador tendem a criar relações mais formais com seus alunos a fim de que possam agir de forma imparcial.

O Perfil 2, denominado Compreensivo, agrupou professores que costumam utilizar em suas aulas estratégias práticas e conhecidas para ensinar. Demonstram preferência para estruturar suas aulas a partir do material didático adotado pela escola. Neste sentido, evidencia-se uma tendência a ser mais tradicional, valorizando informações mais factuais, bem como listas de exercícios e questionários, atividades consideradas por professores com este perfil, como mais práticas, dada a facilidade para serem planejadas com antecedência e corrigidas com maior facilidade, além de poderem ser utilizadas como material de estudo pelos alunos.

A facilidade para se colocar no lugar do outro permite ao professor Compreensivo estabelecer com 
seus alunos relações mais próximas e acolhedoras, incentivando um clima de harmonia e cooperação em suas aulas. Quando precisa decidir, o professor Compreensivo baseia-se em sentimentos, bem como no esforço e no potencial do aluno.

O professor Compreensivo tende a ser meticuloso em seus planejamentos, apresentados aos alunos no início do período letivo. Todo este cuidado com registros e cronogramas pode sinalizar uma dificuldade para lidar com imprevistos, atrasos ou mudanças de última hora nas tarefas propostas. Por ser mais reservado, o professor Compreensivo sente necessidade de ficar um pouco sozinho, principalmente nos momentos em que elabora suas aulas e atividades.

\section{Discussão}

Esta pesquisa teve como objetivo a construção de um instrumento de autorrelato intitulado "Inventário de Estilos de Temperamento do Professor" - IETP. Para tanto foram realizados dois estudos. O primeiro deles avaliou as evidências de validade baseadas no conteúdo dos itens do Inventário. O segundo avaliou as evidências de validade baseadas na estrutura interna do IETP.

A construção do IETP teve como referência os seguintes instrumentos: Student Styles Questionnarie (Oakland, Glutting \& Horton, 1996), e o "Inventário de Estilos de Temperamento para Adultos" (Oakland \& Wechsler, 2014), ambos baseados no MBTI (MyersBriggs Type Indicator), cujo referencial estruturante é a Teoria dos Tipos Psicológicos de Jung. Em comum, os três instrumentos encontram-se estruturados por meio de quatro traços apresentados de forma bipolar para a avaliação do temperamento (Lee, Oakland, \& Ahn, 2010).

Para a elaboração dos itens do IETP foram utilizadas situações do cotidiano escolar associadas às quatro dimensões de estilos de temperamento, sendo que para cada uma delas foram elaborados 10 itens. A cada item foram relacionadas duas alternativas de resposta (A e B), cada uma representando, de maneira oposta, uma das categorias da dimensão mensurada. Portanto, o IETP contém 40 itens dicotômicos de escolha forçada que foram validados pelos cinco juízes que participaram do Estudo 1.

A partir dos resultados apresentados no Estudo 2 nota-se que a dimensão que discriminou o estilo de temperamento do professor foi Processamento da Informação, composta pelos fatores Imaginativo e Prático. Esta condição é diferente do que foi previsto teórica e empiricamente pelos instrumentos referência, onde a direção da energia psíquica, associada por Jung aos tipos Extrovertido ou Introvertido, são considerados os fatores discriminantes do temperamento (Ramos, 2005). A classificação das diferenças individuais é complementada pela associação das funções psicológicas, definidas como aspectos que indicam as preferências do indivíduo para perceber e processar informações - Sensação e Intuição, bem como os parâmetros utilizados na tomada de decisão - Pensamento e Sentimento (Jung, 2011/1921). À estas três dimensões, Myers e Briggs acrescentaram uma quarta para medir como as pessoas se posicionam no ambiente: Julgar e Perceber (Classen, Nichols, McPeek, \& Breiner, 2011; Lara et al., 2012; Oakland \& Hatzchristou, 2010), redefinidas por Oakland como Organizado e Flexível (Oakland, Glutting, \& Horton, 1996).

Considerando que o IETP é um instrumento criado para identificar o perfil profissional de professores, esta solução mostra-se coerente à natureza deste ofício: desenvolver o potencial de alunos a partir da aprendizagem formal. Este resultado também está em sintonia com as várias iniciativas que vem sendo divulgadas por órgãos oficiais, fundações e organizações não governamentais que têm como objetivo oportunizar o desenvolvimento profissional dos docentes que atuam na Educação Básica, tanto os iniciantes quanto os mais experientes. Entretanto, para que possam ser criadas situações de aprendizagem que atendam à demanda atual onde os conhecimentos devem ser utilizados como meio para que os estudantes aprendam a aprender, se comunicar e conviver (Albuquerque, 2010) é preciso primeiro que cada professor possa identificar como reage habitualmente às demandas do ambiente em que trabalha. Em seguida, é preciso que cada docente avalie sua forma de agir a fim de que possa perceber em que medida seus comportamentos facilitam ou dificultam seu trabalho. Esta sua apreciação poderá permitir a identificação de atitudes que poderão ser modificadas ou fortalecidas de acordo com o contexto em que se encontra (Wechsler, 2008), bem como a forma como cada professor elabora suas próprias experiências (Ávila, 2008), condições associadas ao desenvolvimento e à saúde mental de uma pessoa (Klein \& Linhares, 2007, 2010).

Cabe aqui ressaltar que o cotidiano escolar é repleto de exigências que são externas ao professor, tais como conteúdos programáticos, estratégias de ensino, recursos pedagógicos, avaliações e questões relacionadas ao desempenho dos alunos. Todas estas questões são cruciais aos processos de ensino e de aprendizagem, mas, acabam dificultando ao professor voltar sua atenção para si mesmo. Ou seja, em meio às várias exigências, parece não haver espaço para que o 
professor possa reconhecer e refletir sobre sua maneira preferencial de reagir aos estímulos de seu ambiente de trabalho (Albuquerque, 2010), a fim de que possa identificar a forma mais apropriada para utilizar suas habilidades no enfrentamento dos desafios de sala de aula. Dessa forma, cada professor poderá atribuir às metodologias e recursos instrucionais um significado próprio, que vai além do enfoque técnico sobre como de utilizá-los. Abre-se, portanto, um espaço para que os procedimentos apresentados em reuniões de formação possam ser personalizados, deixando de ter um uso instrumental para serem selecionados estrategicamente de acordo com as preferências de cada professor, bem como das necessidades dos alunos e dos recursos disponíveis.

Este exercício de autoconhecimento proposto pelo IETP pode ser enriquecido quando são consideradas as características mais intuitivas presentes na categoria Imaginativo, associadas à Introversão e à percepção dos processos interiores (Jung, 2011/1921). Segundo o referido autor, a intuição fornece dados que podem ser muito importantes para que uma pessoa possa compreender o que ocorre à sua volta. Esta forma de percepção também pode ajudar a prever, de forma mais detalhada, novas possibilidades, bem como qual delas terá mais chances de acontecer. Esta habilidade de antecipação poderá ser desenvolvida na medida em que o professor consiga estabelecer uma ligação entre si próprio e o fenômeno observado, o que pressupõe maior contato com seu mundo interior. Isto pode ser um indício de que, apesar dos dados indicarem uma preponderância na amostra professores com Estilo de Temperamento Catalisador, onde está presente a categoria Extrovertido, considera-se que haja um continuum entre este polo e o Introvertido, o que, de acordo com Jung (2011/1921) indica que uma pessoa pode apresentar comportamentos que variem entre os dois extremos na busca pelo equilíbrio das forças (condição de saúde prevista por Galeno).

Considerando que os professores são os principais articuladores dos processos de ensino e de aprendizagem, oportunizar uma postura mais reflexiva pode incentivá-los a repensar a relação que tem consigo mesmo, com o outro e com o mundo (Cattani, 2010), fortalecendo assim, sua identidade profissional. Esta condição poderá ajudá-los a lidar, por exemplo, com as pressões para cumprir o programa estabelecido e preparar os alunos para avaliações oficiais, podendo abrir espaço para que a sala de aula venha a tornarse um lugar onde podem ser promovidas diferentes possibilidades de aprendizagem.

A descrição e associação dos dois perfis configurados aos estilos de temperamento permitiram identificar também referências que poderão ser utilizadas pelo professor para alinhar melhor seu estilo de ensinar aos diferentes estilos de aprender de seus alunos, possibilitando assim que conheçam outras formas de abordar os conhecimentos trabalhados em sala (Zhang, 2011). Ao valorizar estas características essenciais, mas nem sempre consideradas, os professores poderão melhorar a qualidade do processoensino aprendizagem, não apenas por conta de um maior envolvimento dos alunos, mas também porque as intervenções para contornar as dificuldades percebidas poderão ser mais efetivas (Dinçol et al., 2011; Franzoni \& Assar, 2009).

\section{Limitações e Sugestões para Novas Pesquisas}

O Inventário de Estilos de Temperamento do Professor (IETP) teve comprovadas as evidências de validade baseadas no conteúdo de seus itens. Os resultados do Estudo 1 indicam que a categorização dos itens obteve um índice de concordância entre $80 \%$ e $100 \%$ entre o modelo proposto teoricamente e a apresentada pelos juízes a partir das quatro etapas de avaliação, condição que atesta que os itens representam as categorias avaliadas (Pasquali, 2007). Os resultados do Estudo 2 indicam que foram demonstradas relações de similaridade entre as quatro dimensões que constituem os estilos de temperamento do professor a partir da configuração de dois perfis. Estas condições atestam as evidências de validade baseadas na estrutura interna do IETP (Hair et al., 2009).

O IETP tem como objetivos propor ao professor um exercício de autoconhecimento e de ressignificação de suas práticas. No entanto, para que estas metas possam ser plenamente atingidas, é preciso considerar algumas limitações identificadas nesta pesquisa, que apontam caminhos para estudos futuros que poderão aprimorar o Inventário de Estilos de Temperamento do Professor.

A primeira limitação deste estudo está associada à configuração de apenas dois perfis de estilos de temperamento do professor. Este resultado pode estar vinculado à homogeneidade da amostra de professores, composta por 400 professores que lecionavam no Ensino Fundamental II e no Ensino Médio em escolas públicas. Assim sendo, sugere-se que em estudos futuros sejam incluídos na amostra um maior número de professores que atuam em escolas particulares, bem como professores de outras cidades e regiões do país. Estas novas condições poderão permitir investigar as influências culturais e ambientais no estilo de temperamento do professor. Poderão ser realizados também estudos com professores polivalentes, que 
atuam na Educação Infantil e no Ensino Fundamental I para investigar se os estilos de temperamento do professor podem estar associados à fase de ensino em que leciona.

Estudos futuros poderão também comparar o IETP com outros instrumentos de personalidade. Outros estudos poderão incluir a avaliação que alunos fazem de seus professores, bem como a comparação entre o temperamento de professores e o temperamento de crianças, utilizando para isso, por exemplo, o questionário de Oakland.

Enfim, a validação científica do IETP demonstra que os objetivos propostos na presente pesquisa foram alcançados. Novas possibilidades se configuram para que este instrumento possa ser acessível aos professores visto ser preciso criar um espaço para que possam investir em seu autoconhecimento. Ao conseguir identificar seu modo preferencial de processar as informações, decidir, organizar-se e obter energia para realizar suas tarefas, o docente poderá avaliar mais claramente seus pontos fortes e as necessidades de aprimoramento. Neste processo de descoberta, reflexão e aprendizagem, o docente terá a oportunidade de fortalecer sua prática pedagógica, atribuindo sentido às metodologias que utiliza. Estes podem ser passos valiosos no processo de construção e fortalecimento de sua identidade profissional, bem como de seu estilo pessoal de ensinar, um processo de individuação em busca de sua autorrealização, e não apenas do cumprimento de metas.

\section{Referências}

Albuquerque, C. (2010). Processo Ensino-Aprendizagem: Características do Professor Eficaz. Millenium, 39, 55-71.

Armstrong, S. J., Peterson, E. R., \& Rayner, S. G. (2011). Understanding and defining "cognitive style" and "learning style": a Delphi study in the context of Educational Psychology. Educational Studies, 38(4), 449-455. http://dx.doi. org/10.1080/03055698.2011.643110

Ávila, F. T. P. (2008). Estilos de Ense-anza de los Profesores de la Carrera de Psicología. REMO, V(13), 17-24.

Bayly, B. \& Gartstein, M. (2013). Mother's and father's reports on their child's temperament: Does gender matter? Infant Behavior \& Development, 36(2013), 171-175. http://dx.doi.org/10.1016/j.infbeh.2012.10.008

Borgobello, A., Peralta, N., \& Roselli, N. (2010). El estilo docente universitario en relación al tipo de clase y a la disciplina ense-ada. Libertabit, 16(1), 7-16.

Callueng, C. \& Oakland, T. (2014). If you Do Not Know The Child's Temperament You Do Not Know the Child. Estudos de Psicologia (Campinas), 31(1), 03-13. http://dx.doi.org/10.1590/0103-166X2014000100001

Canals, J., Hernández-Martínez, C., \& Fernández-Ballart, J. D. (2011). Relationships between early behavioural characteristics and temperament at 6 years. Infant Behavior \& Development Journal, 34, 152-160. http://dx.doi. org/10.1016/j.infbeh.2010.11.003

Cattani, D. (2010). Por uma pedagogia de pesquisa educacional e da formação de professores na universidade. Educar em Revista, 37, 77-92. http://dx.doi.org/10.1590/S0104-40602010000200006

Classen, S., Nichols, A. L., McPeek, R., \& Breiner, J. F. (2011). Personality as a predictor of driving performance: an exploratory study. Transportation Research Part F, 14, 381-389. [PSIC - Revista de Psicologia, Vetor Editora, 7(1), 29-38, jan./jun. 2006]. http://dx.doi.org/10.1016/j.trf.2011.04.005

Diçol, S., Temel, S., Oskay, Ö. Ö, Erdogan, U. M., \& Yilmaz, A. (2011). The effect of matching learning styles with teaching styles on success. Procedia Social and Behavioral Sciences, 15, 854-858. http://dx.doi.org/10.1016/j. sbspro.2011.03.198

Evans, C. \& Waring, M. (2009). The place of cognitive style in Pedagogy: realizing potential in practice. In Li-Fang Zhang \& Robert J. Sternberg. Perspectives on the nature of intellectual styles (Cap. 7). New York: Springer Publishing Company.

Frazoni, A. L. \& Assar, S. (2009). Student learning styles adaptation method based on teaching strategies and eletronic media. Educational Technology \& Society, 12(4), 15-29.

Garcia, C. M. (1998). Pesquisa sobre a formação de professores: o conhecimento sobre aprender a ensinar. Revista Brasileira de Educação, 9, 51-75.

Guzzo, R. S. L., Riello, I. C., Primi, R., Serrano, M., Ito, P. C. P., \& Pinho, C. C. M. (2004). Temperamento: Onze anos de levantamento no Psychological Abstracts. Rev. Estudos de Psicologia (Campinas), 21(1), 25-32. http://dx.doi. org/10.1590/s0103-166x2004000100002

Hair, J. F., Black, B., Babin, B. J., Anderson, R. E., \& Tatham, R.L. (2009). Análise Multivariada de Dados. Trad. Adonai Schlup Sant'Anna. Porto Alegre: Bookman.

Ito, P. C. P. \& Guzzo, R. S. L. (2002). Temperamento: Características e determinação genética. Psicologia: Reflexão e Critica, 15(2), 425-436. http://dx.doi.org/10.1590/s0102-79722002000200019 
Joyce, D. (2010). Essentials of Temperament Assessment. Hoboken, New Jersey: John Wiley \& Sons, Inc.

Jung, C. G. (2011). Tipos Psicológicos (4ae ed.). Trad. Lúcia Mathilde Endlich Orth Petrópolis: Vozes.

Klein, V. C. \& Linhares, M. B. M. (2007). Temperamento, comportamento e experiência dolorosa na trajetória de desenvolvimento da criança. Paidéia, 17(36), 33-44.

Klein, V. C. \& Linhares, M. B. M. (2010). Temperamento e Desenvolvimento da Criança: Revisão Sistemática da Literatura. Psicologia em Estudo, 15(4), 821-829. http://dx.doi.org/10.1590/S1413-73722010000400018

Lara, D. R., Bisol, L. W., Brunstein, M. G., Reppold, C. T., Carvalho, H. W. \& Ottoni, G. L. (2012). The Affective and Emotional Composite Temperament (AFECT) model and scale: a system-based integrative approach. Journal of Affective Disorders, 140, 14-37. http://dx.doi.org/10.1016/j.jad.2011.08.036

Lee, D.H., Oakland, T. \& Ahn, C. (2010). Temperament Styles of Children in South Korea and the United States. School Psychology International, 31(1), 77-94. http://dx.doi.org/10.1177/0143034309341621

Lima, L., Lemos, M.S. \& Guerra, M.P. (2010). Adaptação do Inventário de Temperamento para Crianças em Idade Escolar - School-Age Temperament Inventory - SATI, de McClowry a uma população portuguesa. Psicologia, Saúde \& Doenças, 11(1), 55-70.

Lorenzo-Seva, U. \& Ferrando, P. J. (2006). FACTOR: A computer program to fit the exploratory factor analisys model. Behavior Research Methods, 38(1), 88-91. http://dx.doi.org/10.3758/BF03192753

McMahon, T. (2006). Teaching for more effective learning: seven maxims for practice. Radiography, 12, 34-44. http:// dx.doi.org/10.1016/j.radi.2005.03.009

Muritiba, P. M., Casado, T., \& Muritiba, S. N. (2010). Personalidade e preferencia por métodos de ensino: um estudo com graduandos em Administração. R. Adm. FACES Journal, 9(2), 65-85.

Nakano, T. C., Santos, E., Zavarize, S. F., Wechsler, S. M., \& Martins, E. (2010). Estilos de pensar e criarem universitários das áreas de humanas e sociais aplicadas: diferenças por gênero e curso. Psicologia: Teoria e Prática, 12(3), 120-134.

Oakland, T., Glutting, J. J., \& Horton, C. (1996). Student Styles Questionnaire. The Psychological Corporation, USA.

Oakland, T., Pretorius, J. D., \& Lee, D. H. (2008). Temperament Styles of Children from South Africa and the United States. School Psychology International, 29(5), 627-639. http://dx.doi.org/10.1177/0143034308099205

Oakland, T. \& Hatzichristou, C. (2010). Temperament Styles Of Greek and US Children. School Psychology International, 31(4), 422-437. http://dx.doi.org/10.1177/0143034310377302

Pasquali, L. (2007). Validade dos Testes Psicológicos: Será possível reencontrar o caminho? Psicologia: Teoria e Pesquisa, 23(n. esp.), 099-107.

Pinho, C. C. M. (2005). Taxonomia Brasileira da Personalidade: um estudo dos adjetivos da Lingua Portuguesa. [Dissertação de Mestrado], PUC-Campinas.

Pitta, K. B., Santos, L. A. D., Escher, C.A., \& Bariani, I.C.D. (2000). Estilos cognitivos de estudantes de psicologia: impacto da experiência em iniciação científica. Psicologia Escolar e Educacional, 4(2), 41-49. http://dx.doi. org/10.1590/S1413-85572000000200005

Primi, R., Wechsler, S. M., Nakano, T. C., Oakland, T., \& Guzzo, R. S. L. (2014). Using Item Response Theory Methods with the Brazilian Temperament Scale for Students. Journal of Psychoeducational Assessment, 32, 651-662. http:// dx.doi.org/10.1177/0734282914528613

Quintana, T. \& Mu-oz, G. (2010). Validación y adaptación del cuestionario de temperamento y carácter, JTCI de R. Cloninger, versión ni-os y padres, a escolares chilenos entre 8 y 13 a-os de edad. Terapia Psicológica, 28(1), 37-43. http://dx.doi.org/10.4067/S0718-48082010000100004

Ramos, L. M. A. (2005). Tipos Psicológicos na Psicologia Analítica de Carl Gustav Jung. Educação Temática Digital, 4(2), 137-180.

Reali, A. M. M. R., Tancredi, R. M. S. P., \& Mizukami, M. G. N. (2008). Programa de mentoria online: espaço para o desenvolvimento profissional de professoras iniciantes e experientes. Educação e Pesquisa, 34(1), 077-095. http:// dx.doi.org/10.1590/s1517-97022008000100006

Runco, M. (2007). Creativity: Theories and Themes - Research, Development and Practice. Burlington, MA: Elsevier Academic Press.

Sadler-Smith, E. (2009). A duplex model of cognitive style. In Li-Fang Zhang \& Robert J. Sternberg. Perspectives on the nature of cognitive styles. New York: Springer Publishing Company.

Shiner, R. L. (2011). O impacto do temperamento no desenvolvimento infantil: comentários sobre Rothbart, Kagan e Eisenberg. In Tremblay, R. E., Boivin, M., Peters, R. D., \& V. (Eds.). Enciclopédia sobre o Desenvolvimento na Primeira Infância [online]. Montreal, Quebec: Centre of Excellence for Early Childhood Development. Disponível em: http://www.enciclopedia-crianca.com/documents/ShinerPRTxp1.pdf. Consultado em: 26/03/2013.

Sternberg, R. J. \& Grigorenko, E. L. (1997). Are Cognitive Styles Still in Style? American Psychologist, 52(7), 700-712. http://dx.doi.org/10.1037/0003-066X.52.7.700

Sternberg, R.J. \& Grigorenko, E.L. (2001). A capsule history and research on styles. In R. J. Sternberg \& L. F. Zhang (Eds.). Perspectives on thinking, learning and cognitive styles (pp. 1-22). London: Lawerence Erlbaum Associates Publishers. 
Wechsler, S. M. (2008). Estilos de pensar e criar: impacto nas áreas educacional e profissional. Psicodebate, 7, 207-218.

Wechsler, S. M. (2009). Age and gender impact on Thinking and Creating Styles. European Journal of Educational and Psychology, 2(1), 37-48.

Wechsler, S. M., Benson, N., Oakland, T., \& Lourençoni, M. A. (2014). Factorial Structure of the Inventory of Adult Temperament Styles. Psicologia: Reflexão e Crítica, 27(4), 720-727. http://dx.doi.org/10.1590/1678-7153.201427412

Zhang, L. F. \& Sternberg, R. J. (2005). A Threefold Model of Intellectual Styles. Educational Psychology Review, 17(1), 1-53. http://dx.doi.org/10.1007/s10648-005-1635-4

Zhang, L. F. (2011). Teaching styles and conceptions of effective teachers: Tibetan and Han Chinese academics compared. Learning and Individual Differences, 21, 619-623. http://dx.doi.org/10.1016/j.lindif.2011.06.005

Autores:

Cristina Maria D’Antona Bachert - Doutora em Psicologia, PUC Campinas.

Solange Muglia Wechsler - PhD pela University of Georgia (USA).

Wagner de Lara Machado - Doutor em Psicologia, PUC Campinas.

Endereço para correspondência:

Cristina Maria D Antona Bachert

Rua Augusto Lippel, 179 - casa 39

18048-130 Sorocaba, SP, Brasil

<cristinabachert@gmail.com>

Recebido em: 25.03 .2015

Aceito em: 19.01.2016 